\title{
Assessment of cerebral blood flow in patients with multiple chemical sensitivity using near-infrared spectroscopy-recovery after olfactory stimulation: a case-control study
}

\author{
Kenichi Azuma • Iwao Uchiyama • Mari Tanigawa • \\ Ikuko Bamba • Michiyo Azuma • Hirohisa Takano • \\ Toshikazu Yoshikawa · Kou Sakabe
}

Received: 26 December 2014/ Accepted: 4 February 2015/Published online: 15 February 2015

(C) The Japanese Society for Hygiene 2015

\begin{abstract}
Objectives Multiple chemical sensitivity (MCS) is a chronic acquired disorder characterized by non-specific symptoms in multiple organ systems associated with exposure to odorous chemicals. We previously observed significant activations in the prefrontal cortex (PFC) during olfactory stimulation using several different odorants in patients with MCS by near-infrared spectroscopy (NIRS) imaging. We also observed that the patients with MCS did not adequately distinguish non-odorant in the late stage of the repeated olfactory stimulation test. The sensory
\end{abstract}

K. Azuma $(\bowtie) \cdot$ I. Bamba

Department of Environmental Medicine and Behavioral Science,

Kinki University Faculty of Medicine, Osakasayama,

Osaka 589-8511, Japan

e-mail: kenazuma@med.kindai.ac.jp

I. Bamba

e-mail: i-banba@med.kindai.ac.jp

K. Azuma · I. Uchiyama

Sick-house Medical Science Laboratory, Division of Basic

Research, Louis Pasteur Center for Medical Research,

Kyoto 606-8225, Japan

I. Uchiyama

Outpatient Department of Sick-house Syndrome, Hyakumanben

Clinic, Kyoto, Japan

e-mail: iwao-u@cyber.ocn.ne.jp

M. Tanigawa

Clinical Immune Function Laboratory, Division of Basic

Research, Louis Pasteur Center for Medical Research,

Kyoto 606-8225, Japan

e-mail: maritanigawa@louis-pasteur.or.jp

M. Tanigawa

Division of Internal Medicine, Hyakumanben Clinic, Kyoto,

Japan recovery of the olfactory system in the patients with MCS may process odors differently from healthy subjects after olfactory stimulation.

Methods We examined the recovery process of regional cerebral blood flow (rCBF) after olfactory stimulation in patients with MCS. NIRS imaging was performed in 6 patients with MCS and in 6 controls. The olfactory stimulation test was continuously repeated 10 times. The study also included a subjective assessment of the physical and psychological status and of the perception of irritating and hedonic odors.

\author{
M. Azuma \\ Department of Human Environmental Design, Faculty of Health \\ Science, Kio University, Kitakatsuragi-gun, Nara 635-0832, \\ Japan \\ e-mail: m.azuma@kio.ac.jp \\ H. Takano \\ Department of Environmental Engineering, Graduate School of \\ Engineering, Kyoto University, Kyoto 615-8530, Japan \\ e-mail: htakano@health.env.kyoto-u.ac.jp \\ T. Yoshikawa \\ Kyoto Prefectural University of Medicine, Kyoto 602-8566, \\ Japan \\ e-mail: toshi@koto.kpu-m.ac.jp \\ K. Sakabe \\ Department of Anatomy and Cellular Biology, Tokai University \\ School of Medicine, Isehara, Kanagawa 259-1193, Japan \\ e-mail: sakabek@tokai-u.jp
}


Results After olfactory stimulation, significant activations were observed in the PFC of patients with MCS on both the right and left sides compared with controls. The activations were specifically strong in the orbitofrontal cortex (OFC). Compared with controls, autonomic perception and feelings identification were poorer in patients with MCS. OFC is associated with stimuli response and the representation of preferences.

Conclusions These results suggest that a past strong exposure to hazardous chemicals activates the PFC during olfactory stimuli in patients with MCS, and a strong activation in the OFC remains after the stimuli.

Keywords Cerebral blood flow - Multiple chemical sensitivity · Near-infrared spectroscopy · Olfactory stimulation · Orbitofrontal cortex · Recovery

\section{Introduction}

Multiple chemical sensitivity (MCS) is a chronic acquired disorder characterized by non-specific and recurrent multisystem symptoms associated with exposure to common odorous chemicals such as organic solvents, pesticides, cleaning products, perfumes, environmental tobacco smoke, or combustion products [1-3]. According to population-based surveys, the prevalence of MCS is estimated to range from 8 to $33 \%$ [4-9]. Thus, MCS has become a large public health concern during the past two decades, particularly in industrialized countries. The symptoms of MCS can be mild to disabling, and they are triggered by multiple chemicals. These symptoms are reactions to previous chemical exposure that recur on subsequent exposure to the same or structurally unrelated chemicals at levels below those established as having harmful effects in the general population [2]. Central nervous system (CNS) symptoms such as headaches, dizziness, extreme fatigue, and concentration difficulties are common; airway and gastro-intestinal tract symptoms are also frequently reported [2, 10-12]. Diagnosis of MCS can be difficult because of the inability to assess the causal relation between exposure and symptoms [3, 13]. No standardized objective measures to identify MCS and no precise definition of this disorder have been established. Most definitions of MCS are qualitative, relying on subjective reports from patients and clinicians of distressing symptoms and environmental exposure [14].

We previously conducted a near-infrared spectroscopy (NIRS) activation study on olfactory stimulation in patients with MCS [14]. Activation was defined as a significant increase in the regional cerebral blood flow (rCBF) following odorant stimulation. Changes in the blood flow and oxygenation to the brain are closely linked to neural activity [15]. NIRS has been commonly applied in studies of prefrontal activity [16, 17] and is suitable for detecting oxygenation changes in higher cortical regions. Our previous study identified acute activation in the prefrontal cortex (PFC) during olfactory stimulation with several different odorants in patients with MCS [14]. The prefrontal area connects to the anterior cingulate cortex (ACC), an area of odorant-related activation in patients with MCS [18]. The results of challenge tests by exposure to odorous chemicals indicated a neuro-cognitive impairment in patients with MCS, and using single photon-emission computed tomography, brain dysfunction was found particularly in odor-processing areas, thereby suggesting a neurogenic origin of MCS [19]. One possibility is that patients with MCS may have an enhanced top-down regulation of odor response via the cingulate cortex. These findings also suggest that prefrontal information processing associated with the odor-processing neuronal circuits and memory from a past experience of chemical exposure may play significant roles in the pathology of this disorder.

Our previous study also showed that the patients with MCS adequately distinguished non-odorant in 10 odor repetitions during the early stage but not in the late stage of the olfactory stimulation test when the olfactory stimulation test was continuously repeated 10 times. Repeated or prolonged exposure to an odorant typically leads to a stimulus-specific decrease in olfactory sensitivity to that odorant, but sensitivity recovers over time in the absence of further exposure [20]. Thus, we postulate that prefrontal information processing in patients with MCS is activated by an emotional response to a repeated olfactory stimulation in the late stage of the test, and that the processing system in the PFC cannot respond adequately. Further, the sensory recovery of the olfactory system in patients with MCS may process odors differently from healthy subjects after olfactory stimulation. Although recovery is generally evident after short olfactory stimulation on the several tens of second time scale $[21,22]$, the recovery process of patients with MCS may differ from that of healthy subjects. In this study, we examined the recovery process after short olfactory stimulation in patients with MCS, using NIRS imaging.

\section{Methods}

Patients

Patients with MCS were diagnosed in the outpatient department for people with chemical sensitivities in the Hyakumanben Clinic (Outpatient Department of Sick House Syndrome) between October 2009 and January 2013. The same case definitions for MCS (inclusion and 
exclusion criteria), as in our previous study [14], were applied in this study. MCS was diagnosed according to the 1999 consensus criteria [23]. Patients diagnosed with chronic fatigue syndrome, fibromyalgia syndrome, or mental health disorders were excluded from the study. Patients who had hyperpiesia, hyperlipidemia, diabetes, and/or allergic rhinitis were also excluded. Recruitment for this study was conducted 3 months prior to the olfactory stimulation test using NIRS. The MCS condition of all patients was confirmed by the clinic physician during recruitment. Controls were recruited and selected to match the patients by age and sex at the group level. The same inclusion and exclusion criteria were applied for all patients and controls as those in our previous study [14]. Inclusion was based on the scores of the Quick Environmental Exposure and Sensitivity Inventory (QEESI), whereas exclusion criteria included abnormal hematological examinations, smoking, drug or alcohol abuse, medications, pregnancy, and severe nasal stuffiness.

This study was approved by the ethical committee for human research at the Hyakumanben Clinic (99642-61) and the Louis Pasteur Centre for Medical Research (LPC.11) and was performed according to the guidelines of the Declaration of Helsinki (1975). All patients provided written informed consent and received the equivalent of 5000 JPY for their participation. This study was conducted from November 2012 to March 2013.

\section{Olfactory stimulation}

The same card-type olfactory identification test kit (Open Essence; Wako Pure Chemical Industries, Ltd., Osaka, Japan) and odorants [mandarin orange (MO), Japanese cypress (JC), menthol (Mt), and perfume (Pf)] as those in our previous study were used for the olfactory stimulation test [14]. Perception of these odors was tested by placing the card at approximately $30 \mathrm{~mm}$ from the noses of both patients with MCS and controls.

\section{Experimental procedure}

In the present study, we followed the same experimental procedure as that in our previous study [14] and added a recovery period after the olfactory stimuli. Interviews were conducted immediately before the olfactory stimulation test and the assessments of health and nasal symptoms. The test room was maintained at a temperature of approximately $23{ }^{\circ} \mathrm{C}$. Patients sat in a comfortable chair and remained in the test room long enough to feel comfortable before being exposed to the odorants. During the experiments, the patients closed their eyes and slowly repeated the Japanese alphabet in an undertone to establish a stable rCBF prior to the olfactory stimulation. They

\begin{tabular}{|c|c|c|c|c|c|c|}
\hline \multirow[b]{2}{*}{ Time (sec) } & \multicolumn{3}{|c|}{$\begin{array}{c}\text { Stimulus } \\
\text { MO (1) }\end{array}$} & \multicolumn{2}{|c|}{$\begin{array}{l}\text { Response to } \\
\text { questionnaire }\end{array}$} & $\begin{array}{r}\text { Stimult } \\
\text { Pf (2) }\end{array}$ \\
\hline & 30 & 10 & $\mathbf{3 0}$ & 30 & 30 & 10 \\
\hline & $\begin{array}{l}\text { Pre-r } \\
\text { (Basel }\end{array}$ & & ecov & & Basel & \\
\hline
\end{tabular}

Fig. 1 Experimental protocol. First, the subjects had a 30-s pre-rest. Then, the subjects were given an olfactory stimulus for $10 \mathrm{~s}$, followed by a 30 -s post-rest and 30-s self-reporting to a questionnaire on irritating and hedonic scales, respectively. The 100-s cycles per odorant were repeated 10 times in a row

stopped repeating the Japanese alphabet and closed their eyes during the olfactory stimulation, which lasted for $10 \mathrm{~s}$. Olfactory stimulation was performed after a 30 -s pre-rest period to establish the baseline level (Fig. 1).

The questionnaire on irritating and hedonic scales was completed immediately after a 30 -s rest period (post-rest) to allow recovery after the olfactory stimulation. The response time for the questionnaire was secured for $30 \mathrm{~s}$. After that, the same process was repeated for an additional 9 olfactory stimuli. Irritation was evaluated on a visual analogue scale, with responses ranging from "not at all" to "strong". Hedonic responses were rated on a 5-point Likert scale ranging from pleasant (1) to unpleasant (5).

Olfactory stimuli were applied in the following order: MO, Pf, non-odorant (NO), JC, Mt, Pf, JC, NO, Mt, and MO. The order of the first block was as follows: MO (1), Pf (2), NO (3), JC (4), and Mt (5). The order of the second block was permuted from the first block as follows: Pf (6), JC (7), NO (8), Mt (9), and MO (10). The 100-s cycles were repeated 10 times in a row. Thus, the order of 10 repetitions (1-10) was as follows: MO (1), Pf (2), NO (3), JC (4), Mt (5), Pf (6), JC (7), NO (8), Mt (9), and MO (10).

\section{NIRS data acquisition}

NIRS works on the principle that near-infrared light is absorbed by oxygenated (oxyHb) and deoxygenated (deoxyHb) hemoglobin $(\mathrm{Hb})$ but not by other tissues. Changes in oxyHb concentration in the PFC were measured using the functional NIRS topography system OMM-3000 Optical Multi-channel Monitor (Shimadzu Corporation, Kyoto, Japan). These changes reflect neuronal activity as their levels correlate with evoked changes in $\mathrm{rCBF}[15,24$, 25]. When neurons become active, local blood flow to the relevant brain regions increases and oxygenated blood displaces deoxygenated blood. Measurement of oxyHb concentrations is most useful because changes in oxyHb are the most sensitive indicators of changes in rCBF among the three NIRS parameters (oxyHb, deoxyHb, and totalHb) $[26,27]$. Pairs of illuminators and detectors were set $3 \mathrm{~cm}$ apart in a $3 \times 9$ lattice pattern to form 42 channels through a holder set in the PFC. Changes in the oxyHb concentration were recorded every $130 \mathrm{~ms}$ using the NIRS 
system. Optical data were analyzed on the basis of the modified Beer-Lambert Law and signals reflecting the oxyHb concentration changes in an arbitrary unit were calculated (millimolar-millimeter) [14].

Questionnaire on physical and psychological status

Patients completed a self-report questionnaire for the assessment of physical and psychological parameters, which included the Chemical Sensitivity Scale for Sensory Hyper-reactivity (CSS-SHR) [28], the Somato-Sensory Amplification Scale (SSAS) [29], the Autonomic Perception Questionnaire (APQ) [30], the Tellegen Absorption Scale (TAS) [31], the Marlowe-Crowne Social Desirability Scale [32], the Taylor Manifest Anxiety Scale (TMAS) [33], the Negative Affectivity Scale (NAS) [34], and the Toronto Alexithymia Scale (TAS-20) that evaluates the total score and the scores of the three subscales, which assess difficulties in identifying feelings (DIF), difficulties in describing feelings (DDF), and externally-oriented thinking (EOT) [35].

\section{Statistical analyses}

To assess the recovery status after the olfactory stimulation, the oxyHb concentrations between the 30 -s rest period after the olfactory stimulation and the baseline during the pre-rest period were compared in each channel. Because raw data of NIRS provided only relative values and could not be averaged directly across patients or compared among channels, raw data from each channel were converted into $z$-scores $[14,36-38]$. In the present study, we used the same statistical analyses as that in our previous study [14]. The $t$ test was used to compare the brain activity obtained from NIRS imaging for each channel between cases and controls. The non-parametric Mann-Whitney $U$ test was used to analyze the results of the olfactory stimulation questionnaire and to quantify the differences between patients with MCS and controls. The $t$ test was applied to analyze the results of the physical and psychological scales to determine differences between patients with MCS and controls at baseline. All data analyses were performed using the SPSS statistics software, version 22.

\section{Results}

\section{Participants}

Participants included 10 patients with MCS (age, 28-64 years; mean, $51.0 \pm 10.6$ years; all females) and six controls (age, 36-58 years; mean, $45.7 \pm 8.3$ years; all females). Three patients with MCS did not fulfill the inclusion criteria of QEESI. The fourth patient with MCS had severe allergenic reactions to allergens of cedar pollen, mites, cats, dogs, and fungi, and showed a high value of immunoglobulin $\mathrm{E}$ type on hematological examinations. The remaining six non-smoking patients with MCS (age, 49-64 years; mean, $54.5 \pm 5.9$ years; all females) and six non-smoking controls (age, 36-58 years; mean, $45.7 \pm 8.3$ years; all females) passed all criteria and were included in the analyses. All six patients with MCS had participated in our previous study [14]. Of the six controls, two controls had participated in our previous study [14] and the remaining four controls were participating for the first time in the present study. All patients with MCS tried to avoid the exposure to odorous chemicals as much as possible. These patients were homemakers or pensioners and their occupational histories showed that previous occupations included a clerical employee (office or retail store), a fabric tinter, and a supermarket baker. Two controls also tried to avoid exposure to odorous chemicals as much as possible. Their occupations were teacher. Four controls did not consciously try to avoid exposure to odorous chemicals, and their occupations were as follows: teacher, office worker, child welfare volunteer, and the fourth was a homemaker whose previous occupation was in sales for a general insurance company.

\section{NIRS imaging and subjective evaluation of odors}

Time-course of average $z$-scores of all channels for oxyHb in the patients with MCS group and controls during prerest, stimulus, and post-rest are shown in Fig. 2. Results of the $t$ test in terms of the average of all channels (1-42) comparing $z$-scores for oxyHb concentrations between patients with MCS and controls are shown in Table 1. The first olfactory stimulation with MO (1) lead to increased rCBF levels in the PFC, which was not significantly different between patients with MCS and controls.

Increases in $\mathrm{rCBF}$ levels in patients with MCS were suppressed after exposure to the non-odorant tests NO (3) and NO (8) on the third repetition. There was no difference between patients with MCS and controls in the PFC responses. Significant differences in the PFC responses were observed between patients with MCS and controls after Pf (2), JC (4), Mt (5), Pf (6), Mt (9), and MO (10) olfactory stimuli. The increases in the PFC response after the olfactory stimulation were significant in patients with MCS. No significant difference was observed in the JC (7) test, but as shown in Fig. 2, MCS patients had increases in rCBF levels during $10 \mathrm{~s}$ after the stimulus, which were not observed in the control group.

Table 2 shows the correlation coefficient between rCBF after the first and second exposure to the same odor in terms of $z$-scores for all channels (1-42). Comparing the 
Fig. 2 Time-course of average $z$-scores of all channels for oxyHb in patients with MCS $(n=6)$ and controls $(n=6)$ during pre-rest (baseline, 10-30 s), stimulus (30-40 s), and post-rest (recovery, $40-70 \mathrm{~s}) . Y$ - and $X$-axes represent $z$-scored oxyHb values and times. Law and signals reflecting the oxyHb

concentration changes in an arbitrary unit were calculated (millimolar-millimeter). Data of the signal were adjusted by an FFT (Fast Fourier

Transform) filter smoothing technique (OriginPro 9.1 software of OriginLab Corporation). The cutoff frequency was determined at thirty-five points. MCS group is indicated as a black line and control is indicated as gray line. $M O$ mandarin orange, $P f$ perfume, $N O$ non-odorant, $J C$ Japanese cypress, $M t$ menthol. Numbers in parentheses indicate the orders of the 10 repetitions $(1-10)$

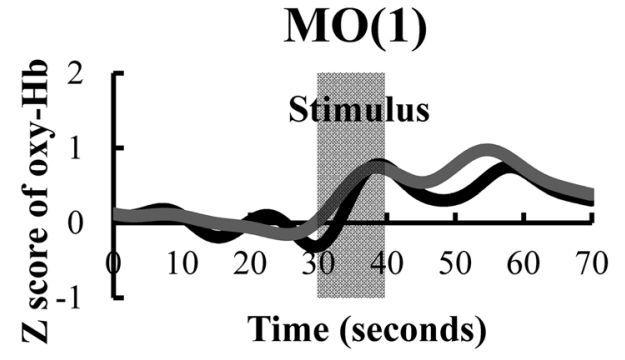

$\operatorname{Pf}(2)$

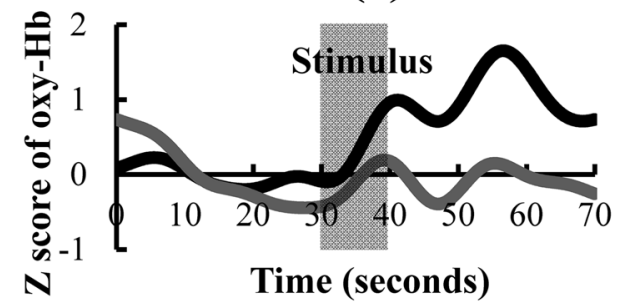

NO(3)

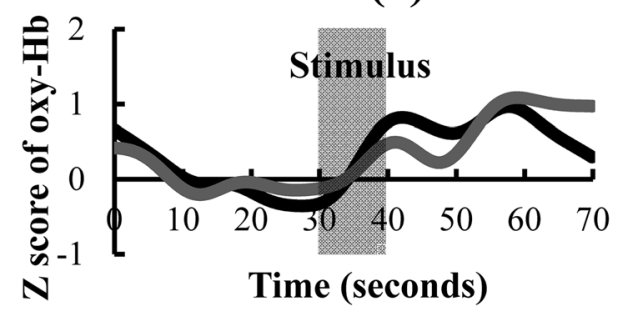

JC(4)

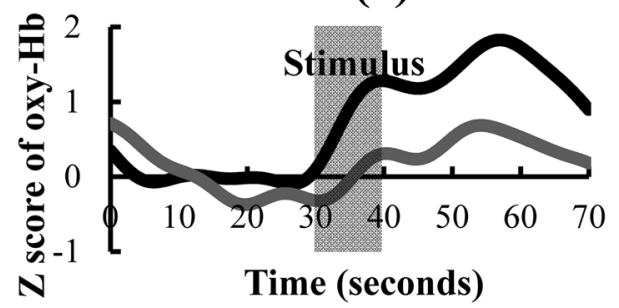

$\operatorname{Mt}(5)$

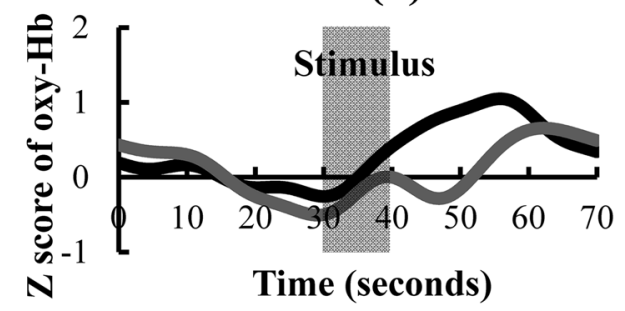

-MCS Control

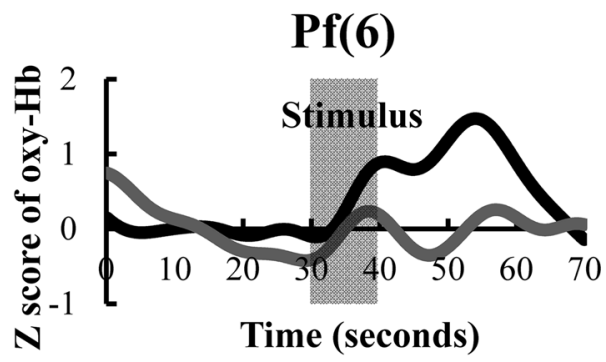

JC(7)
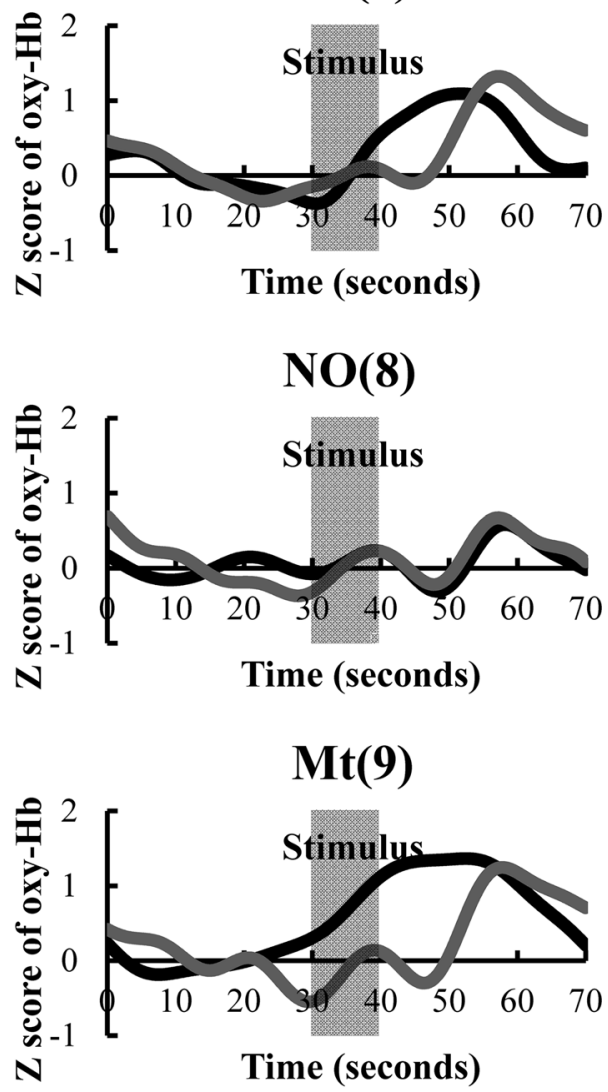

MO(10)

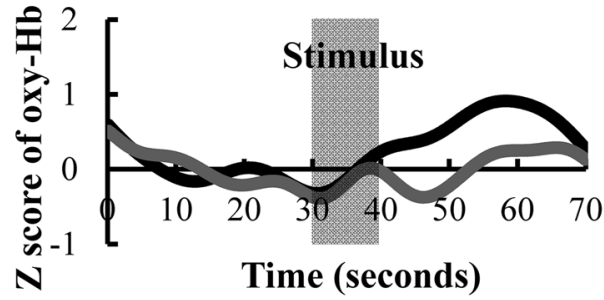

rCBF between the first and second exposures revealed significant correlations in both patients with MCS and controls for all stimuli, with the exception of MO and NO in patients with MCS. The correlation coefficients of patients with MCS were lower overall than those of controls. As in our previous study [14], the variation within the MCS group was larger than in the control group. In the subjective evaluation, both patients with MCS and controls 
Table 1 The $t$ test results in terms of average values for all channels (1-42) comparing $z$-scores for oxyHb between patients with MCS and controls

\begin{tabular}{llcc}
\hline Test & MCS $(n=6)$ & Controls $(n=6)$ & $p$ value \\
\hline MO (1) & $0.47(2.40)$ & $0.66(2.05)$ & 0.336 \\
Pf (2) & $1.05(2.57)$ & $-0.11(1.33)$ & $<0.001^{*}$ \\
NO (3) & $0.70(2.47)$ & $0.73(1.48)$ & 0.895 \\
JC (4) & $1.41(3.37)$ & $0.40(1.35)$ & $<0.001^{*}$ \\
Mt (5) & $0.73(3.02)$ & $0.23(1.08)$ & $0.013^{*}$ \\
Pf (6) & $0.82(3.13)$ & $-0.03(1.02)$ & $<0.001^{*}$ \\
JC (7) & $0.66(3.11)$ & $0.63(2.63)$ & 0.905 \\
NO (8) & $0.13(1.15)$ & $0.23(1.34)$ & 0.407 \\
Mt (9) & $1.06(2.93)$ & $0.56(2.50)$ & $0.038^{*}$ \\
MO (10) & $0.61(1.59)$ & $0.01(0.94)$ & $<0.001^{*}$ \\
\hline
\end{tabular}

Values are expressed as means ( \pm standard deviations). Numbers in parentheses in column 1 indicate the order of the 10 repetitions (1-10)

$M O$ mandarin orange, $P f$ perfume, $N O$ non-odorant, JC Japanese cypress, $M t$ menthol

* Significant at $p<0.05$

Table 2 Correlation coefficient $(r)$ between $\mathrm{rCBF}$ after the first and second exposures to the odor in terms of $z$-scores for all channels $(1-42)$

\begin{tabular}{|c|c|c|c|c|}
\hline \multirow[t]{2}{*}{ Odorant } & \multicolumn{2}{|c|}{$\operatorname{MCS}(n=6)$} & \multicolumn{2}{|c|}{ Controls $(n=6)$} \\
\hline & $r$ & $p$ value & $r$ & $p$ value \\
\hline MO & 0.082 & 0.197 & 0.193 & $0.002 *$ \\
\hline Pf & 0.321 & $<0.001 *$ & 0.548 & $<0.001 *$ \\
\hline $\mathrm{NO}$ & 0.092 & 0.144 & 0.447 & $<0.001 *$ \\
\hline $\mathrm{JC}$ & 0.250 & $<0.001 *$ & 0.479 & $<0.001 *$ \\
\hline Mt & 0.302 & $<0.001 *$ & 0.400 & $<0.001 *$ \\
\hline
\end{tabular}

Values are expressed as Pearson product-moment correlation coefficients

$M O$ mandarin orange, $P f$ perfume, $N O$ non-odorant, JC Japanese cypress, $M t$ menthol

* Significant at $p<0.05$

responded "not at all" on the irritation scale and "undecided" on the hedonic scale for NO (Fig. 3). The results of the hedonic scale indicated that scores of most patients with MCS were significantly higher than those of controls. The results of the irritation scale indicated that the Pf (2) and MO (10) scores of patients with MCS were significantly higher than those of controls. Scores for MO, Pf, $\mathrm{JC}$, and Mt were also higher in the patients with MCS than in controls, but the differences were not statistically significant. Large ranges of scores in patients with MCS and controls were assumed to be causally related to the results.

Figure 4 provides the topographical maps of average $z$ scores for oxyHb in patients with MCS and controls. Figure 5 shows the average $t$ values for each channel
A

MCS $\square$ Controls

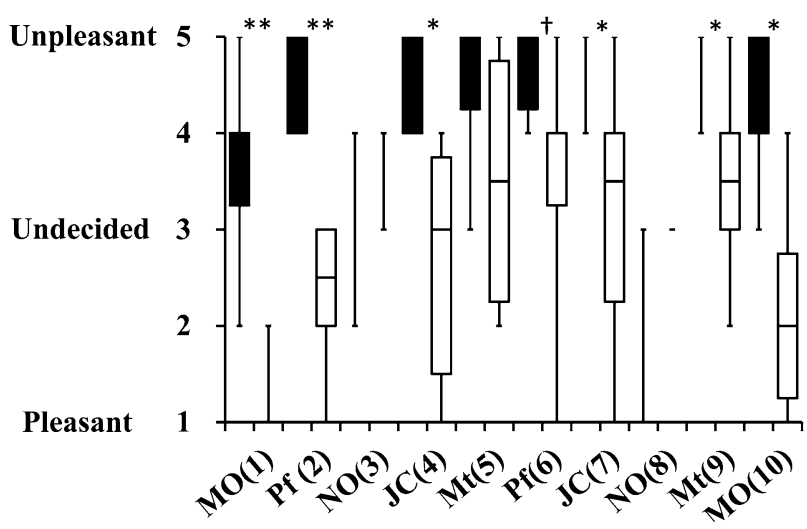

B

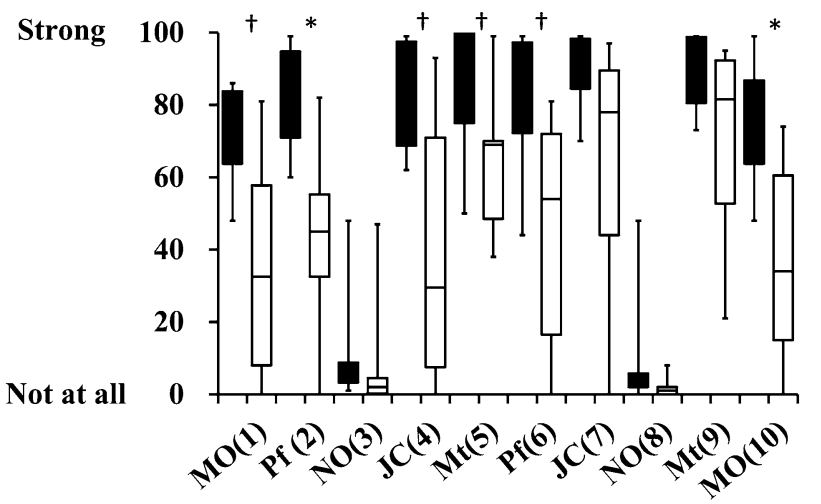

Fig. 3 Ratings of hedonic (a) and irritating (b) odors by patients with MCS $(n=6)$ and controls $(n=6)$ after the olfactory stimulation. $M O$ mandarin orange, $P f$ perfume, $N O$ non-odorant, JC Japanese cypress, $M t$ menthol. Numbers in parentheses indicate orders of the 10 repetitions (1 to 10). Statistically significant differences between groups are indicated. ${ }^{*} p<0.05,{ }^{*} p<0.01$. Significant tendencies are indicated: ${ }^{\dagger} p<0.10$

comparing the $z$-scores for oxyHb between patients with MCS and controls. Even after olfactory stimuli, significant activations were observed in the PFC of patients with MCS on both right and left sides (distinct from the center of the PFC) compared with controls. Activation was defined as a significant increase in rCBF due to olfactory stimulation. The activations were especially strong in the lateral orbitofrontal cortex (OFC), on both the right and left sides of the OFC in the PFC (Fig. 4). These remaining activations after the olfactory stimuli were stronger in the test for $\mathrm{Pf}$ (2) on the second repetition, for JC (4) on the fourth repetition, for $\mathrm{Mt}$ (5) on the fifth repetition, and for Pf (6) on the sixth repetition. After the test for Pf (6), the regional differences of the activation area between patients with MCS and controls were decreased. Our previous study suggested that the olfactory system in patients with MCS could not adequately process odors in the late stage of the 


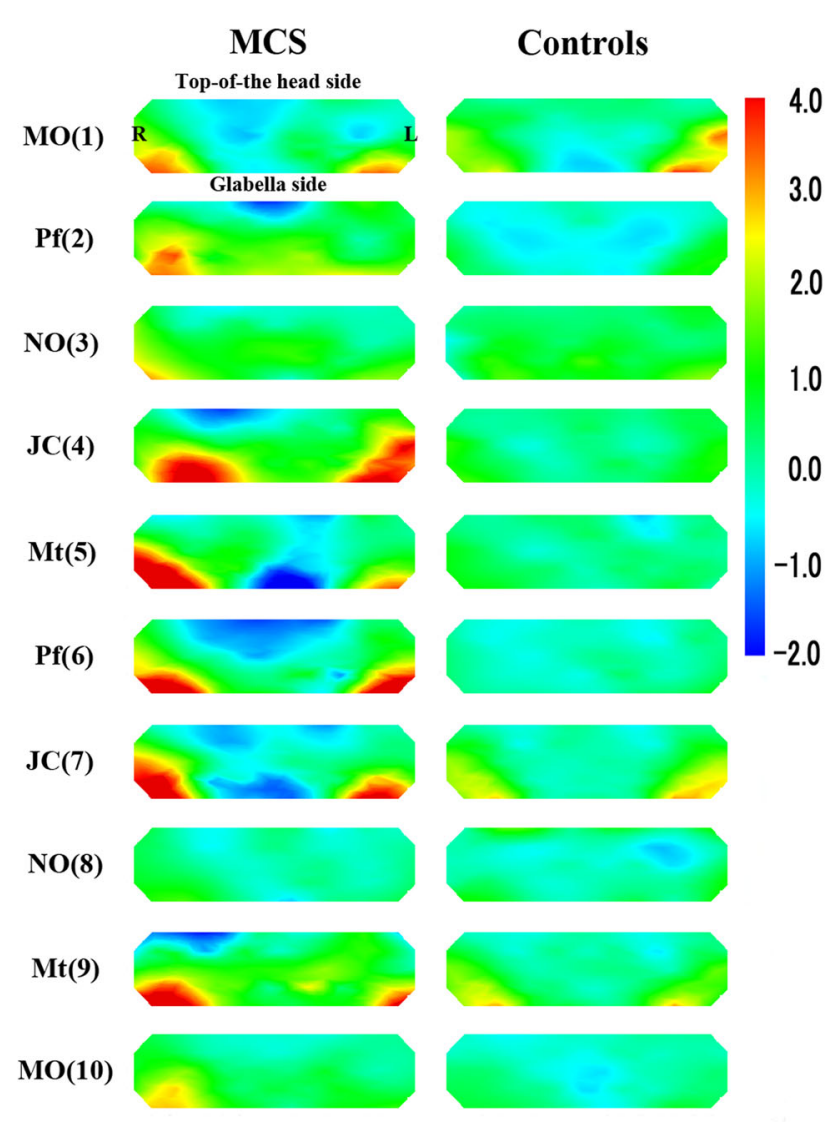

Fig. 4 Topographical maps of average $z$-scores for oxyHb between patients with MCS $(n=6)$ and controls $(n=6)$. MO mandarin orange, $P f$ perfume, $N O$ non-odorant, $J C$ Japanese cypress, $M t$ menthol. Numbers in parentheses indicate the order of the 10 repetitions $(1-10)$

olfactory stimulation test. And then, the NIRS imaging revealed that the CNS of patients with MCS may have been confused in the late stage of the olfactory stimulation test [14]. The results of the present study support this hypothesis.

Physical and psychological measurements

Table 3 shows the results of the $t$ test for the physical and psychological scales. CSS-SHR scores were significantly higher of patients with MCS than of controls $(p<0.001)$. Thus, chemical sensitivity in patients with MCS was demonstrated not only by the results of QEESI but also by those of the CSS-SHR scale. In the psychological evaluations, the APQ $(p<0.1)$ and TAS-20 DIF $(p<0.1)$ scores showed a higher tendency only for patients with MCS compared with controls, probably because of the small sample size, but these differences were significant in our previous study [14]. No significant differences were observed in the SSAS, TAS, MCSD, TMAS, TAS-20 total, TAS-20 DDF, and TAS-20 EOT scores, similar to our previous study [14].

\section{Discussion}

As shown in Fig. 2, strong activation was observed during olfactory stimulation with several different odorants in patients with MCS compared with controls. All patients with MCS participating in this study had participated in our previous study [14]. Although the previous study had been conducted 1-2 years before this study, similar responses were observed in both.

The first olfactory stimulation with MO (1) lead to increased $\mathrm{rCBF}$ levels in the PFC, which was not significantly different between patients with MCS and controls. Because MO (1) was the first test, the patients may not have had the chance to get used to the olfactory stimuli and the response may have been caused by affective tension. After the first test, rCBF levels in controls remained almost stable until the end of the test involving MO (10). Thus, we did not superimpose the data of first and second block but showed all the data as the results.

In the subjective measurement by irritating and hedonic scales and physical and psychological measurements, the overall results were similar to our previous study [14] and were reproducible. In the present study, even after olfactory stimulation, significant activations were observed in the PFC of patients with MCS than in that of controls. The activations were especially strong on both right and left sides of OFC in the PFC. Further, the activations in both patients with MCS and controls were suppressed after the olfactory stimulation involving NO, and the differences were not significant. These results indicate that the olfactory system in patients with MCS adequately distinguishes the non-odorant. Comparing the rCBF between the first and second exposures revealed significant correlations in both patients with MCS and controls for all stimuli, with the exception of MO and NO in patients with MCS. The lack of correlation in the MO and NO test in patients with MCS may be due to the small sample size.

An odorant-related increase in activation in the ACC has been observed in patients with MCS [18]. The ACC is involved in adequate control of top-down or bottom-up modulation of stimuli and is connected to the PFC. Past exposures to hazardous chemicals are stored as memories in the PFC through olfactory nerve circuits, causing various physical or psychological responses such as emotional, visceral, or autonomic responses during the processing of top-down stimuli when exposed to odorants later in life [14]. In the present study, we found that recovery from activation in the PFC after an olfactory stimulation is delayed in patients with MCS compared with controls. These findings support the current understanding of the pathology of this disorder: compared to healthy subjects, patients with MCS strongly respond to odorants that they encounter in daily life, the repeated daily exposure to the odorants keeps 


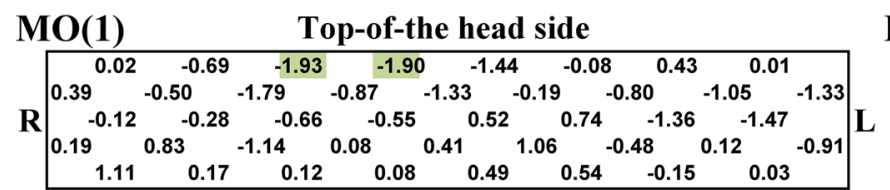

$\operatorname{Pf}(2)$

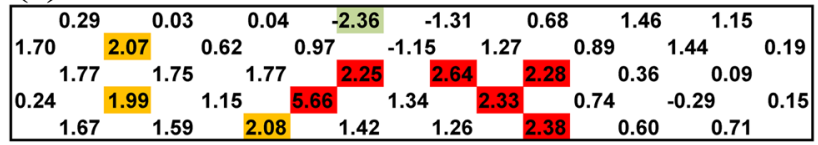

NO(3)

\begin{tabular}{|cccccccccc|}
\hline 0.65 & -0.76 & -1.79 & -1.33 & -1.23 & -1.03 & -1.18 & -0.69 & \\
0.83 & -0.34 & -0.97 & 0.47 & -1.58 & 0.51 & -0.80 & -0.72 & -0.86 \\
0.36 & 0.040 & 0.61 & 0.25 & 0.40 & 0.85 & -0.76 & -1.18 & \\
0.46 & 0.34 & 0.29 & 0.29 & -0.34 & 0.37 & 0.54 & -0.61 & -0.13 & 0.06 \\
0.53 & 0.44 & 0.46 & -0.54 & -1.09 & -0.56 & -0.11 & 0.41 & \\
\hline
\end{tabular}

\section{$\mathrm{JC}(4)$}

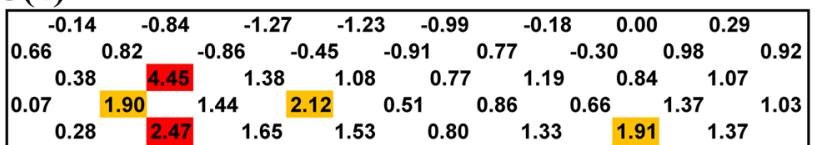

\section{$\operatorname{Mt}(5)$}

\begin{tabular}{|c|c|c|c|c|c|c|c|c|c|}
\hline-0.97 & -1.18 & -0.46 & -0.64 & -2.41 & -0.1 & & 0.52 & \multicolumn{2}{|c|}{-0.25} \\
\hline .72 & 0.72 & -0.16 & 0.53 & -0.80 & -1.15 & 0.47 & & -0.24 & -0.0 \\
\hline 1.57 & 0.58 & 1.30 & 0.97 & -1.21 & -1 & & 0.22 & 2. & \\
\hline 87 & 2.02 & 1.17 & 1.27 & -0.93 & -0.9 & -0.60 & & & 2.01 \\
\hline
\end{tabular}

$\operatorname{Pf}(6)$

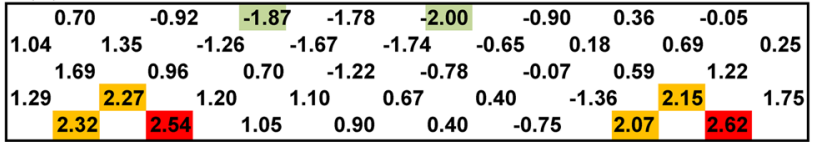

\section{$\mathrm{JC}(7)$}

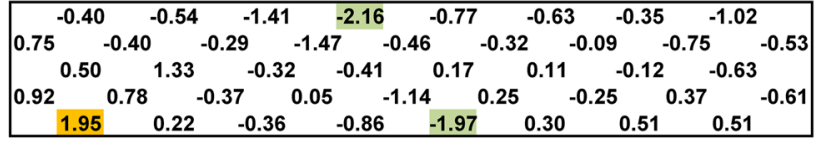

\section{NO(8)}

\begin{tabular}{|cccccccccc|}
\hline 1.03 & 1.04 & 0.20 & & -0.13 & 0.32 & 1.08 & 0.34 & 0.55 & -1.77 \\
0.02 & 0.72 & -0.36 & -0.47 & 0.75 & -0.48 & 0.54 & -0.38 & -0.66 \\
0.02 & 0.33 & 0.18 & -0.14 & -0.06 & 0.14 & -0.90 & -1.68 & \\
-0.52 & -0.03 & 0.45 & 0.01 & -0.40 & -0.51 & 0.05 & -1.89 & -0.95 \\
-0.24 & 0.14 & 0.41 & -0.81 & -1.55 & 0.19 & -1.00 & -1.12 & \\
\hline
\end{tabular}

\section{$\operatorname{Mt}(9)$}

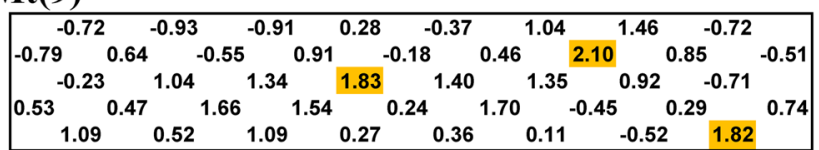

\section{MO(10)}

\begin{tabular}{|ccccccccccc|}
\hline & 1.40 & 2.03 & 0.68 & -0.19 & -0.04 & -0.78 & 0.02 & -0.03 & \\
1.75 & & 1.26 & & 0.58 & 0.41 & 0.43 & -0.22 & 1.18 & 0.04 & 0.61 \\
0.90 & & 1.24 & 1.09 & 1.12 & 1.34 & 1.44 & -0.03 & -0.44 & \\
0.69 & 1.28 & & 1.53 & 0.28 & 1.52 & 0.68 & 1.74 & 0.03 & 0.32 \\
1.48 & 1.86 & 1.15 & 0.65 & 0.77 & 1.48 & 0.22 & 1.10 & \\
\hline
\end{tabular}

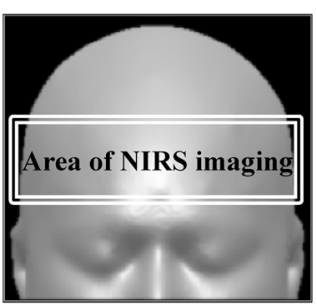

Fig. 5 Average $t$ value of each channel comparing $z$-scores for oxyHb between patients with MCS $(n=6)$ and controls $(n=6)$. Red rectangles denote statistically significant positive correlations $(p<0.05)$, and blue rectangles denote statistically significant

them in a reactive state. Due to their physical and psychological intolerance to odorants, the patients try to avoid exposure to the odorants. In this study, 4 patients with MCS had episodes of initial exposure to chemicals that triggered the first symptoms. These included organic solvents or incense at the workplace, exhaust gas from diesel machines in the neighborhood, odors from pesticides, or fragrance from a neighbor. Two patients had episodes of repeated exposure to solvents emitted from a neighboring industrial plant or a neighboring paint store, respectively. Patients with MCS complained about a chemical-sensitive condition thereafter. The psychological evaluations in our study support the theory of a strong response in patients with MCS.

In the recovery stage after the stimulation, the activation was especially strong in the OFC. The olfactory neuroanatomy is intertwined, via extensive reciprocal axonal negative correlations $(p<0.05)$. Yellow rectangles denote positive correlations $(p<0.10)$, and green rectangles denote negative correlations $(p<0.10)$. The channels are located in the position shown by the white double line rectangle below

connections, with primary emotion areas including the amygdala, hippocampus, and OFC [39, 40]. Olfactory stimulation directly activates amygdala neurons, innervating a region in the OFC. The olfactory sense has a unique intimacy with the emotion system, and the perception of smell is known to be dominated by emotion [41]. Strong activation in the OFC might remain as potent affective experiences following olfactory stimuli in patients with MCS compared with controls. In this study, lateral orbitofrontal regions were specifically activated in the patients with MCS. The valence of odors is represented in particular in the OFC [42]. Nearly all odors were evaluated as unpleasant by the MCS patients in the subjective evaluation after the stimulation. Pleasant odors preferentially activate medial orbitofrontal regions, whereas unpleasant odors activate more lateral regions [43-46]. The strong activations of the lateral $\mathrm{OFC}$ in the patients with 
Table 3 Results of the $t$ test for the physical and psychological scales

\begin{tabular}{lccc}
\hline Scales & MCS $(n=6)$ & Controls $(n=6)$ & $p$ value \\
\hline QEESI (CI) & $75.0(19.2)$ & $4.2(5.3)$ & $<0.001^{*}$ \\
QEESI (OI) & $33.2(24.1)$ & $6.7(15.8)$ & $0.048^{*}$ \\
QEESI (SS) & $72.7(13.5)$ & $5.2(11.3)$ & $<0.001^{*}$ \\
CSS-SHR & $51.7(2.2)$ & $32.5(3.8)$ & $<0.001^{*}$ \\
SSAS & $34.3(8.7)$ & $28.0(5.2)$ & 0.156 \\
APQ & $178.3(39.4)$ & $131.2(42.6)$ & 0.075 \\
TAS & $13.3(7.7)$ & $12.8(10.4)$ & 0.927 \\
MCSD & $16.0(4.7)$ & $20.5(5.1)$ & 0.141 \\
TMAS & $11.0(4.1)$ & $8.2(1.5)$ & 0.156 \\
NAS & $37.0(17.0)$ & $27.5(8.6)$ & 0.250 \\
TAS-20 total & $48.7(16.2)$ & $38.0(4.0)$ & 0.172 \\
TAS-20 DIF & $14.5(5.4)$ & $9.5(3.0)$ & 0.076 \\
TAS-20 DDF & $12.8(4.8)$ & $10.3(1.2)$ & 0.264 \\
TAS-20 EOT & $21.3(7.2)$ & $18.2(4.0)$ & 0.375 \\
\hline
\end{tabular}

Values are expressed as means ( \pm standard deviations)

$C I$ chemical intolerance, $O I$ other intolerance, $S S$ symptom severity

* Significant at $p<0.05$

MCS suggest that these odors were extremely unpleasant for the MCS patients.

Both the ACC and OFC are implicated in decisionmaking, emotion, and social behavior. Recent evidence suggests that the ACC and OFC make distinct contributions to each of these aspects of decision-making [47]. The OFC is involved in the cognitive processing of stimuli and the representation of preferences. The ACC may mediate the relationship between a past experience and the choice of the next action. Thus, our results suggest that a past strong exposure to hazardous chemicals activates the ACC (and the connected PFC) during olfactory stimuli in the patients with MCS, and a strong activation in the OFC remains after the stimuli. In particular, the lateral OFC is specifically activated when the odor is unpleasant for the patients with MCS. However, the OFC and ACC are anatomically interconnected, and their interaction stimulates decisionmaking. Their individual function independent of each other remains unclear. Further research is required to understand the recovery process in MCS and the pathology of this disorder.

The present study has some limitations. First, the very small sample size makes the results vulnerable to selection bias. This could be alleviated by including a larger study population. However, despite the small sample size of this study, differences between the patients with MCS and the controls in the NIRS imaging were evident. The results indicate that the evaluation combining NIRS imaging with olfactory stimulation tests is a valuable method for the objective evaluation of MCS. Second, to the best of our knowledge, this is the first case-control study evaluating changes in $\mathrm{rCBF}$ in the PFC using NIRS imaging after olfactory stimulation in patients with MCS. Further longtime evaluation after olfactory stimulation would provide valuable information for understanding the pathology of MCS. A third limitation of this study is the lack of standardized objective measures to identify and define MCS. Therefore, most definitions of MCS are entirely qualitative, relying on subjective reports of distressing symptoms and environmental exposure from patients and clinicians. Several individuals with self-reported MCS symptoms were excluded, at the discretion of the clinic physician, because of mental disorders or allergic symptoms.

In conclusion, despite the small sample size, this experimental study detected an activation that remained even after olfactory stimulation, specifically in the PFC of patients with MCS. We propose that recovery from such activation is delayed in patients with MCS and that their chemical-sensitive state remains due to the repeated daily exposure, leading them eventually to develop intolerance to these odorants. Our study demonstrates that NIRS imaging objectively reflects the status of patients with MCS.

Acknowledgments This study was financially supported by a Grant-in-Aid for Scientific Research (ID: 22590568) provided by the Japan Ministry of Education, Culture, Sports, Science and Technology. This study was also financially supported by a health science research grant (H25-5962) from the Japan Ministry of the Environment and a Grant-in-Aid for Health and Labour Sciences Research Grant (H25-008) provided by the Japan Ministry of Health, Labour and Welfare. We express our sincere appreciation to the staff members of the Hyakumanben Clinic for their assistance in data collection.

Conflict of interest The authors declare that no conflict of interests exists.

Ethical standard This study was approved by the ethical committee for human research at the Hyakumanben Clinic (99642-61) and the Louis Pasteur Center for Medical Research (LPC.11).

Informed consent This manuscript does not contain the personal medical information about an identifiable living individual. All patients provided written informed consent.

\section{References}

1. Cullen MR. The worker with multiple chemical sensitivities: an overview. Occup Med. 1987;2:655-61.

2. Graveling RA, Pilkington A, George JP, Butler MP, Tannahill SN. A review of multiple chemical sensitivity. Occup Environ Med. 1999;56:73-85.

3. Winder C. Mechanisms of multiple chemical sensitivity. Toxicol Lett. 2002;128:85-97.

4. Berg ND, Linneberg A, Dirksen A, Elberling J. Prevalence of self-reported symptoms and consequences related to inhalation of airborne chemicals in a Danish general population. Int Arch Occup Environ Health. 2008;81:881-7. 
5. Caress SM, Steinemann AC. Prevalence of multiple chemical sensitivities: a population-based study in the southeastern United States. Am J Public Health. 2004;94:746-7.

6. Hausteiner C, Bornschein S, Hansen J, Zilker T, Förstl H. Selfreported chemical sensitivity in Germany: a population-based survey. Int J Hyg Environ Health. 2005;208:271-8.

7. Kreutzer R, Neutra RR, Lashuay N. Prevalence of people reporting sensitivities to chemicals in a population-based survey. Am J Epidemiol. 1999;150:1-12.

8. Meggs WJ, Dunn KA, Bloch RM, Goodman PE, Davidoff AL. Prevalence and nature of allergy and chemical sensitivity in a general population. Arch Environ Health. 1996;51:275-82.

9. Azuma K, Uchiyama I, Katoh T, Ogata H, Arashidani K, Kunugita N. Prevalence and characteristics of chemical intolerance: a Japanese population-based study. Arch Environ Occup Health. 2014;. doi:10.1080/19338244.2014.926855 [Epub ahead of print].

10. Sorg BA. Multiple chemical sensitivity: potential role for neural sensitization. Crit Rev Neurobiol. 1999;13:283-316.

11. Berg ND, Linneberg A, Dirksen A, Elberling J. Phenotypes of individuals affected by airborne chemicals in the general population. Int Arch Occup Environ Health. 2009;82:509-17.

12. Lacour M, Zunder T, Schmidtke K, Vaith P, Scheidt C. Multiple chemical sensitivity syndrome (MCS) - suggestions for an extension of the US MCS-case definition. Int J Hyg Environ Health. 2005;208:141-51.

13. McKeown-Eyssen GE, Baines CJ, Marshall LM, Jazmaji V, Sokoloff ER. Multiple chemical sensitivity: discriminant validity of case definitions. Arch Environ Health. 2001;56:406-12.

14. Azuma K, Uchiyama I, Takano H, Tanigawa M, Azuma M, Bamba I, et al. Changes in cerebral blood flow during olfactory stimulation in patients with multiple chemical sensitivity: a multichannel near-infrared spectroscopic study. PLoS One. 2013;8(11):e80567. doi:10.1371/journal.pone.0080567.

15. Hock C, Müller-Spahn F, Schuh-Hofer S, Hofmann M, Dirnagl $\mathrm{U}$, Villringer A. Age dependency of changes in cerebral hemoglobin oxygenation during brain activation: a near-infrared spectroscopy study. J Cereb Blood Flow Metab. 1995;15:1103-8.

16. Obrig $\mathrm{H}$, Villringer $\mathrm{A}$. Beyond the visible-imaging the human brain with light. J Cereb Blood Flow Metab. 2003;23:1-18.

17. Okamoto M, Matsunami M, Dan H, Kohata T, Kohyama K, Dan I. Prefrontal activity during taste encoding: an fNIRS study. Neuroimage. 2006;31:796-806.

18. Hillert L, Musabasic V, Berglund H, Ciumas C, Savic I. Odor processing in multiple chemical sensitivity. Hum Brain Mapp. 2007;28:172-82.

19. Orriols R, Costa R, Cuberas G, Jacas C, Castell J, Sunyer J. Brain dysfunction in multiple chemical sensitivity. J Neurol Sci. 2009;287:72-8.

20. Dalton P. Psychophysical and behavioral characteristics of olfactory adaptation. Chem Senses. 2000;25:487-92.

21. Elsberg CA, Spotnitz H. Relation of stimulation time of receptors to recovery time in the nervous system: visual, olfactory and auditory senses. J Neurophysiol. 1939;2:227-33.

22. Zufall F, Leinders-Zufall T. The cellular and molecular basis of odor adaptation. Chem Senses. 2000;25:473-81.

23. Anonymous. Multiple chemical sensitivity: a 1999 Consensus. Arch Environ Health. 1999;54:147-49.

24. Kameyama M, Fukuda M, Yamagishi Y, Sato T, Uehara T, Ito M, et al. Frontal lobe function in bipolar disorder: a multichannel near-infrared spectroscopy study. Neuroimage. 2006;29:172-84.

25. Tanida M, Sakatani K, Takano R, Tagai K. Relation between asymmetry of prefrontal cortex activities and the autonomic nervous system during a mental arithmetic task: near infrared spectroscopy study. Neurosci Lett. 2004;369:69-74.
26. Hoshi Y, Kobayashi N, Tamura M. Interpretation of near-infrared spectroscopy signals: a study with a newly developed perfused rat brain model. J Appl Physiol. 2001;90:1657-62.

27. Strangman G, Culver JP, Thompson JH, Boas DA. A quantitative comparison of simultaneous BOLD fMRI and NIRS recordings during functional brain activation. Neuroimage. 2002;17:719-31.

28. Nordin S, Millqvist E, Lowhagen O, Bende M. A short Chemical Sensitivity Scale for assessment of airway sensory hyperreactivity. Int Arch Occup Environ Health. 2004;77:249-54.

29. Barsky AJ, Goodson JD, Lane RS, Cleary PD. The amplification of somatic symptoms. Psychosom Med. 1988;50:510-9.

30. Mandler G, Mandler JM, Uviller ET. Autonomic feedback: the perception of autonomic activity. J Abnorm Psychol. 1958;56: 367-73.

31. Tellegen A, Atkinson G. Openness to absorbing and self-altering experiences ("'absorption"), a trait related to hypnotic susceptibility. J Abnorm Psychol. 1974;83:268-77.

32. Crowne DP, Marlowe D. A new scale of social desirability independent of psychopathology. J Consult Psychol. 1960;24: 349-54.

33. Bendig AW. The development of a short form of the manifest anxiety scale. J Consult Psychol. 1956;20:384.

34. Watson D, Clark LA, Tellegen A. Development and validation of brief measures of positive and negative affect: the PANAS scales. J Pers Soc Psychol. 1988;54:1063-70.

35. Taylor GJ, Bagby RM, Parker JD. The 20-Item Toronto Alexithymia Scale. IV. Reliability and factorial validity in different languages and cultures. J Psychosom Res. 2003;55:277-83.

36. Schroeter ML, Zysset S, Kruggel F, von Cramon DY. Age dependency of the hemodynamic response as measured by functional near-infrared spectroscopy. Neuroimage. 2003;19:555-64.

37. Matsuda G, Hiraki K. Sustained decrease in oxygenated hemoglobin during video games in the dorsal prefrontal cortex: a NIRS study of children. Neuroimage. 2006;29:706-11.

38. Horaguchi T, Ogata Y, Watanabe N, Yamamoto M. Behavioral and near-infrared spectroscopy study of the effects of distance and choice in a number comparison task. Neurosci Res. 2008;61: 294-301.

39. Haberly LB. Parallel-distributed processing in olfactory cortex: new insights from morphological and physiological analysis of neuronal circuitry. Chem Senses. 2001;26:551-76.

40. Gottfried JA. Central mechanisms of odour object perception. Nat Rev Neurosci. 2010;11:628-41.

41. Krusemark EA, Novak LR, Gitelman DR, Li W. When the sense of smell meets emotion: anxiety-state-dependent olfactory processing and neural circuitry adaptation. J Neurosci. 2013;33: 15324-32.

42. de Araujo IE, Rolls ET, Velazco MI, Margot C, Cayeux I. Cognitive modulation of olfactory processing. Neuron. 2005;46: 671-9.

43. Anderson AK, Christoff K, Stappen I, Panitz D, Ghahremani DG, Glover G, et al. Dissociated neural representations of intensity and valence in human olfaction. Nat Neurosci. 2003;6:196-202.

44. Gottfried JA, Dolan RJ. The nose smells what the eye sees: crossmodal visual facilitation of human olfactory perception. Neuron. 2003;39:375-86.

45. Rolls ET, Kringelbach ML, de Araujo IE. Different representations of pleasant and unpleasant odours in the human brain. Eur $\mathbf{J}$ Neurosci. 2003;18:695-703.

46. Zald DH, Pardo JV. Emotion, olfaction, and the human amygdala: amygdala activation during aversive olfactory stimulation. Proc Natl Acad Sci USA. 1997;94:4119-24.

47. Rushworth MF, Behrens TE, Rudebeck PH, Walton ME. Contrasting roles for cingulate and orbitofrontal cortex in decisions and social behaviour. Trends Cogn Sci. 2007;11:168-76. 